\title{
Acute inflammation stimulates a regenerative response in the neonatal mouse heart
}

\author{
Chunyong Han ${ }^{1, *}$, Yu Nie ${ }^{1, *}$, Hong Lian ${ }^{1}$, Rui Liu ${ }^{1}$, Feng He ${ }^{1}$, Huihui Huang ${ }^{1}$, Shengshou Hu ${ }^{1}$ \\ ${ }^{I}$ State Key Laboratory of Cardiovascular Disease, Fuwai Hospital, National Center for Cardiovascular Disease, Chinese Academy \\ of Medical Sciences and Peking Union Medical College, Beijing 100037, China
}

Cardiac injury in neonatal 1-day-old mice stimulates a regenerative response characterized by reactive cardiomyocyte proliferation, which is distinguished from the fibrotic repair process in adults. Acute inflammation occurs immediately after heart injury and has generally been believed to exert a negative effect on heart regeneration by promoting scar formation in adults; however, little is known about the role of acute inflammation in the cardiac regenerative response in neonatal mice. Here, we show that acute inflammation induced cardiomyocyte proliferation after apical intramyocardial microinjection of immunogenic zymosan A particles into the neonatal mouse heart. We also found that cardiac injury-induced regenerative response was suspended after immunosuppression in neonatal mice, and that cardiomyocytes could not be reactivated to proliferate after neonatal heart injury in the absence of interleukin-6 (IL-6). Furthermore, cardiomyocyte-specific deletion of signal transducer and activator of transcription 3 (STAT3), the major downstream effector of IL-6 signaling, decreased reactive cardiomyocyte proliferation after apical resection. Our results indicate that acute inflammation stimulates the regenerative response in neonatal mouse heart, and suggest that modulation of inflammatory signals might have important implications in cardiac regenerative medicine.

Keywords: heart regeneration; inflammation; interleukin-6; STAT3; cell proliferation

Cell Research (2015) 25:1137-1151. doi:10.1038/cr.2015.110; published online 11 September 2015

\section{Introduction}

Cardiomyocyte loss underlies the fundamental cause of heart failure in ischemic heart disease $[1,2]$. Although evidence in support of cardiomyocyte self-renewal in adult hearts exists [3-5], the rate of self-renewal is extremely low, and adult cardiomyocytes cannot mount a profound proliferation response to sufficiently regenerate the lost myocardium after cardiac injury [6, 7]. Cardiac damage in adult mammals typically leads to fibrotic scar formation and contractile dysfunction, with minimal regeneration of the heart muscle $[6,8]$. In contrast, neonatal hearts can regenerate completely within 21 days after

*These two authors contributed equally to this work.

Correspondence: Shengshou $\mathrm{Hu}^{\mathrm{a}}, \mathrm{Yu} \mathrm{Nie}^{\mathrm{b}}$

${ }^{a}$ Tel: +86-10-88396011; Fax: +86-10-88398359

E-mail: shengshouhu@yahoo.com

${ }^{\mathrm{b}}$ Tel: +86-10-60866139; Fax: +86-10-60866092

E-mail: nieyuniverse@126.com

Received 27 January 2015; revised 25 April 2015; accepted 17 July 2015; published online 11 September 2015 the tip of the heart of 1-day-old mice has been resected. This cardiac regenerative response in 1-day-old mice is characterized by reactive cardiomyocyte proliferation [8]. Due to their remarkable capacity for heart regeneration, neonatal mice are an ideal tool for studying mammalian cardiac regeneration.

Previous studies have reported that manipulation of cell-cycle regulators such as cyclin A2, cyclin D1 and cyclin D2 can increase cardiomyocyte number and lead to fibrotic scar reduction and cardiac function improvement in the damaged myocardium of adult mice [9-12]. Several signaling pathways, including the NRG1/ErbB4 [13] and Hippo-Yap pathways [14-16], have been shown to impact cardiomyocyte proliferation and cardiac repair in adult mammals. In addition, microRNA (miRNA)-199a and miRNA-590 were shown to significantly promote cardiomyocyte proliferation in neonates and adults and improve cardiac function in adult mice after myocardial infarction (MI) [17]. Recently, miRNA-195 and Meis1 were identified as inhibitors of neonatal heart regeneration after MI [18-20]. There is also evidence that the 
direct or indirect stimulation of neoangiogenesis by macrophages is required for neonatal heart regeneration after MI [21]; however, the molecular mechanisms by which cardiac injury stimulates the regenerative response in neonatal mice are largely unknown.

Acute inflammation occurs immediately after myocardial injury in neonates and adults [8, 22-24]. Neutrophils and monocytes from the bloodstream are recruited to and infiltrate the injured myocardium to remove cellular debris. A number of cytokines are released to modulate the microenvironment of the injured tissue, and acute cardiac inflammation in adult mice is generally believed to exert a negative effect on heart regeneration by promoting fibrotic scar formation [23-27]. However, several recent studies have suggested positive roles for inflammation in tissue repair [28-32]. These investigations revealed that activated innate immune signals including interleukin-4 and -13 (IL-4/IL-13), and monocytes/macrophages facilitate skeletal muscle regeneration [31, 32]. After MI, the injured myocardium sequentially recruits Ly-6C high and $-6 \mathrm{C}^{\text {low }}$ monocytes, which is a prerequisite for myocardial infarct healing [28-30]. In neonatal mice, a robust acute inflammatory response accompanies the early stages of cardiac regeneration, although the inflammatory response at the injured apical myocardium in neonatal mice does not hamper heart regeneration, as shown previously [8]. However, little is known about the role of acute inflammation in the neonatal cardiac regenerative process.

In the current study, we found that acute cardiac inflammation could induce cardiomyocyte proliferation after apical intramyocardial microinjection of zymosan A (ZA) into neonatal mouse hearts. Moreover, injury-induced cardiomyocyte proliferation was inhibited by immunosuppression. These results indicate that acute cardiac inflammation is required for the cardiac regenerative response after cardiac injury in neonatal mice. Our studies further showed that IL-6 was essential for heart regeneration in neonatal mice. STAT3 is the major downstream effector of IL-6 signaling, and consistently, cell-specific ablation of STAT3 in cardiomyocytes impaired their proliferative response after apical resection (AR) in neonatal mice. Thus, our study reveals an important role of acute inflammation in neonatal cardiac regeneration, and suggests that inflammatory signals might be targeted to promote cardiac regeneration, minimize fibrotic scar formation and ameliorate cardiac function.

\section{Results}

An acute inflammatory response occurs immediately after AR and ZA microinjection of neonatal mouse hearts
To investigate acute inflammation in the injured heart of neonatal mice, we resected the apex of neonatal 1-dayold mouse heart [8] and then performed a cytokine panel array analysis, which showed that several inflammatory cytokines were significantly upregulated in the resected hearts (Supplementary information, Figure S1 and Video S1). Additionally, Ly-6G (1A8) inflammatory leukocytes [33] were rapidly recruited to the injured myocardium (Figure 1A). The inflammatory markers il6, illb and chemokine (C-C motif) ligand 3 (ccl3) were highly expressed, and this elevation continued for 7 days after injury (Figure 1B; $P<0.05$ ).

We also induced a sterile inflammatory response through apical intramyocardial microinjection of ZA in neonatal 1-day-old mice (Figure 1C and 1D). We found that inflammation was significantly induced (Figure 1E and $1 \mathrm{~F}$ ), without influencing cardiac cell viability and size (Supplementary information, Figure S2 and S3) or cardiac tissue integrity disruption (Supplementary information, Figure S4). Similar to AR, ZA microinjection led to the recruitment of Ly-6G (1A8) ${ }^{+}$inflammatory leukocytes (Figure 1E) and significantly elevated the expression of il6, illb and $c c l 3$ (Figure 1F; $P<0.05$ ). This inflammatory response persisted for 7 days (Figure 1F). Thus, ZA microinjection-induced inflammation could recapitulate aspects of acute inflammation in the resected hearts of neonatal 1-day-old mice.

Injured hearts cannot regenerate after immunosuppression in neonatal mice

To test whether acute cardiac inflammation was required for heart regeneration in neonatal mice, we administered dexamethasone (Dex, $0.05 \mathrm{mg} / \mathrm{kg}$ ) via intraperitoneal injection (i.p.) from 0 to 7 days post-resection (dpr) to inhibit the acute inflammatory response in neonatal mice (Figure 2A). The immunosuppressive effects in the resected hearts were confirmed by decreased Ly$6 \mathrm{G}(1 \mathrm{~A} 8)^{+}$leukocyte infiltration and down-regulation of $i l 6, i l l b$ and $c c l 3$ expression in mice treated with AR and Dex (Figure 2B and 2C). Dex treatment alone in uninjured mice did not significantly alter the expression of $i l 6, i l l b$ and $c c l 3$ in the heart (Supplementary information, Figure S5), indicating that Dex treatment impeded only the injury-activated inflammatory response, without any significant effects on constitutive cytokine expression. There were also no significant effects on cardiac cell viability (Supplementary information, Figure S6), cardiomyocyte size (Supplementary information, Figure S7), or body size and heart weight (Supplementary information, Figure S8). Thus, Dex treatment partially blocked the acute inflammatory response after AR in neonatal mouse hearts. 
A

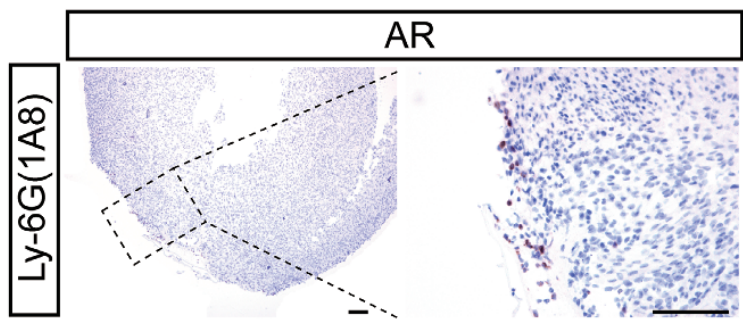

C

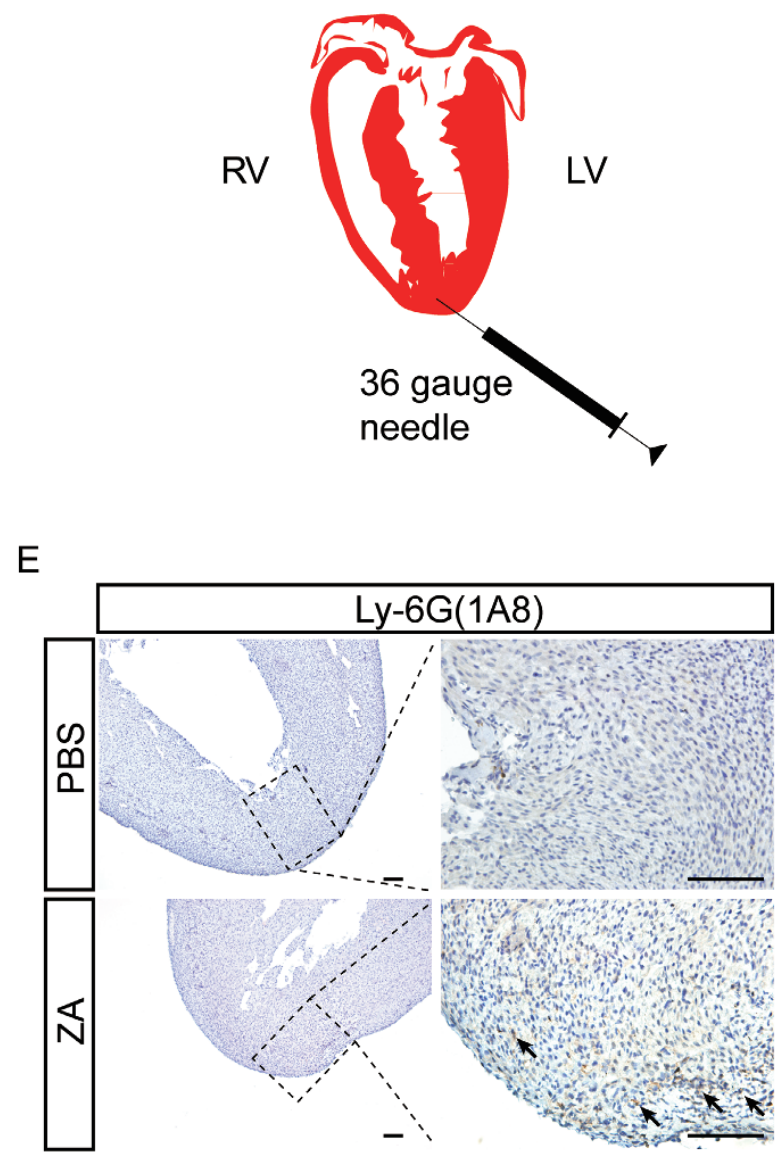

B

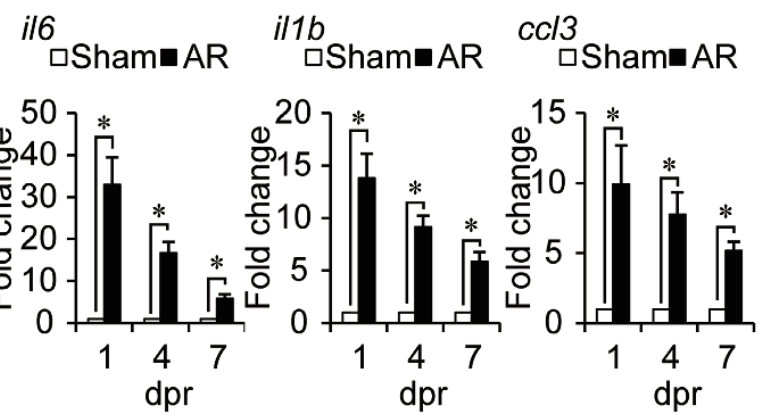

$\mathrm{D}$

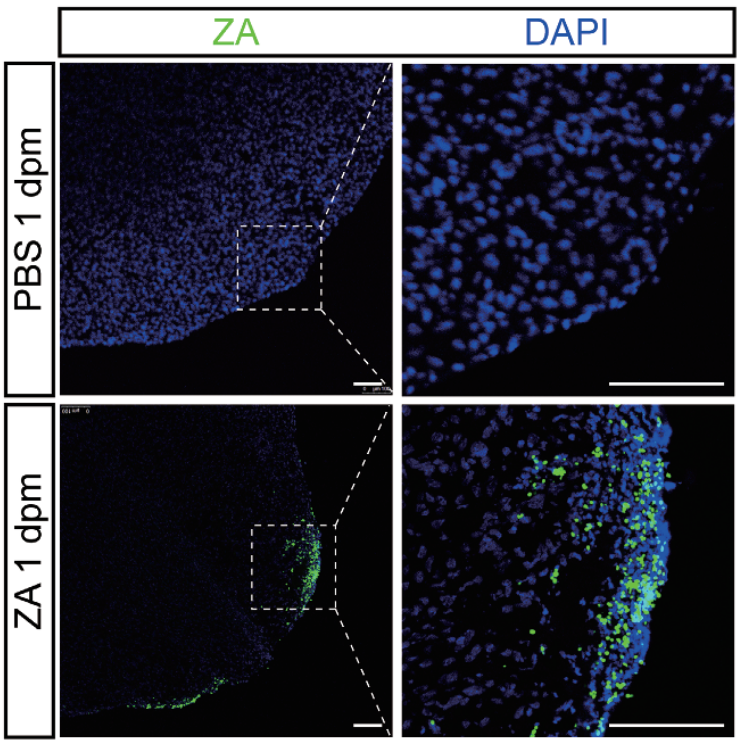

$\mathrm{F}$
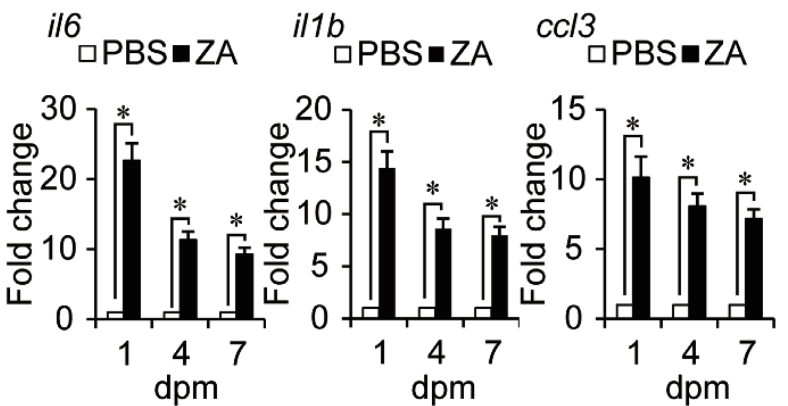

Figure 1 The acute inflammatory response occurs immediately after AR and ZA microinjection in neonatal mouse hearts. (A) Immunostaining for Ly-6G (1A8) at 1 day post-resection (dpr) in the neonatal mouse heart. The high-magnification views of the boxed areas are presented on the right. AR, apical resection; scale bars, $100 \mu \mathrm{m}$. (B) qRT-PCR assays of the expression of inflammatory markers (ill, il/ b and $c c / 3$ ) in sham and AR hearts at 1, 4 and $7 \mathrm{dpr}$. Gene expression in AR hearts is presented as the fold change compared to sham hearts. $n=3$ per group. (C, D) Apical intramyocardial microinjection of ZA into the neonatal 1-day-old mouse heart. ZA was conjugated with Alexa Fluor ${ }^{\circledR} 488$ to track its distribution in the cardiac apex. The high-magnification views of the boxed areas are presented on the right. dpm, days post-microinjection. Scale bars, 100 $\mu \mathrm{m}$. (E) Immunostaining for Ly-6G (1A8) in the PBS and ZA micro-injected apical myocardium at $1 \mathrm{dpm}$ in neonatal mice. The high-magnification views of the boxed areas are presented on the right. Arrows indicate Ly- $6 \mathrm{G}(1 \mathrm{~A} 8)^{+}$leukocytes. Scale bars, $100 \mu \mathrm{m}$. (F) qRT-PCR assays of the expression of inflammatory markers (il6, il1 b and $c c / 3$ ) at 1, 4 and $7 \mathrm{dpm}$. Gene expression in the ZA micro-injected hearts is presented as the fold change compared with PBS micro-injected hearts. $n=3$ per group; values are presented as the mean $\pm \mathrm{SEM} ; * P<0.05$. 
To explore whether acute cardiac inflammation was necessary for heart regeneration, we resected the cardiac apex in immunosuppressed mice. Histological analysis revealed that, compared to heart regeneration in the phosphate-buffered saline (PBS)-treated mice, the immunosuppressed hearts failed to regenerate the lost myocardium at $21 \mathrm{dpr}$. Instead, a fibrotic scar was formed at the cardiac apex (Figure 2D and 2E). The contractile function of the heart was also significantly impaired after immunosuppression at $21 \mathrm{dpr}$ by echocardiographic analysis (Figure 2F). Thus, we conclude that acute inflammation is required for cardiac regeneration in neonatal mice.

Acute inflammatory response is necessary and sufficient for reactive cardiomyocyte proliferation in the neonatal mouse heart

Cardiac injury induced an acute inflammatory response yet promoted cardiomyocyte proliferation in neonatal mice [8]. To investigate whether acute inflammation could independently trigger cardiomyocyte proliferation without apparent cardiac lesions, we performed phospho-histone $\mathrm{H} 3$ (pH3) and Ki67 immunostaining together with $\alpha$-actinin co-localization analysis to detect cardiomyocyte proliferation in PBS and ZA micro-injected hearts. We observed that the ZA-induced inflammation increased the number of $\mathrm{pH}^{+}$cardiomyocytes compared with the PBS micro-injected hearts (PBS: $12.41 \pm 1.6$ versus ZA: $25.32 \pm 1.77$ cardiomyocytes $/ \mathrm{mm}^{2}$ at 4 days post-microinjection (dpm); $P<0.05$ and PBS: $12.27 \pm$ 0.84 versus ZA: $27.45 \pm 2.52$ cardiomyocytes $/ \mathrm{mm}^{2}$ at $7 \mathrm{dpm} ; P<0.05)$. The inflammation also enhanced the number of $\mathrm{Ki} 67^{+}$cardiomyocytes in the ZA micro-injected hearts (PBS: $45.54 \pm 2.15$ versus ZA: $63.23 \pm 2.3$ cardiomyocytes $/ \mathrm{mm}^{2}$ at $4 \mathrm{dpm} ; P<0.05$ and PBS: 40.36 \pm 2.38 versus ZA: $70.07 \pm 3.35$ cardiomyocytes $/ \mathrm{mm}^{2}$ at 7 dpm; $P<0.05$; Figure 3A and Supplementary information, Figure S9). We also performed an apical intramyocardial microinjection of ZA into 7-day-old mouse hearts and then conducted $\mathrm{pH} 3$ and Ki67 staining with $\alpha$-actinin co-immunostaining at 1 and $7 \mathrm{dpm}$. We found that acute inflammation did not induce cardiomyocyte proliferation in 7-day-old mouse hearts (Supplementary information, Figure S10). To determine whether the induced inflammation increased the number of newly formed cardiomyocytes in the neonatal mouse heart, we performed pulsechase labeling of 5-bromo-2-deoxyuridine (Brdu) over a 21-day period after ZA microinjection of neonatal 1-dayold mice. The number of $\mathrm{Brdu}^{+}$cardiomyocytes in the ZA micro-injected hearts was significantly increased compared with the PBS micro-injected hearts (PBS: 63.86 \pm 2.96 versus $Z A: 138.36 \pm 8.3$ cardiomyocytes $/ \mathrm{mm}^{2} ; P<$ 0.05 ) at $21 \mathrm{dpm}$ (Figure 3B). These findings indicate that acute inflammation could induce cardiomyocyte proliferation in neonatal mouse hearts.

To evaluate the effects of immunosuppression on reactive cardiomyocyte proliferation in neonatal mice, we conducted $\mathrm{pH} 3$ and cardiac troponin $\mathrm{T}(\mathrm{cTnT})$ double immunofluorescence staining in Dex-treated hearts after AR. Immunosuppression led to a significant decrease in $\mathrm{pH}^{+}$cardiomyocytes compared with PBS-treated control hearts at $4 \mathrm{dpr}(\mathrm{AR}+\mathrm{PBS}$ : $28.57 \pm 2.14$ versus AR + Dex: $10.84 \pm 1.47$ cardiomyocytes $\left./ \mathrm{mm}^{2} ; P<0.05\right)$ and at $7 \mathrm{dpr}$ (AR + PBS: $25.76 \pm 1.25$ versus AR + Dex: $12.52 \pm 1.08$ cardiomyocytes $\left./ \mathrm{mm}^{2} ; P<0.05\right)$. Moreover, the immunosuppressed hearts contained far fewer Ki67 cardiomyocytes at $4 \mathrm{dpr}(\mathrm{AR}+\mathrm{PBS}: 72.41 \pm 7.44$ versus AR + Dex: $22.98 \pm 1.6$ cardiomyocytes $\left./ \mathrm{mm}^{2} ; P<0.05\right)$ and at $7 \mathrm{dpr}(\mathrm{AR}+\mathrm{PBS}: 81.49 \pm 7.6$ versus AR + Dex: $28.21 \pm 1.68$ cardiomyocytes $/ \mathrm{mm}^{2} ; P<0.05$; Figure 3C and Supplementary information, Figure S11). Additionally, we conducted Brdu pulse-chase labeling of cardiomyocytes in newborn mice and identified a significantly decreased number of $\mathrm{Brdu}^{+}$cardiomyocytes in the immunosuppressed hearts (AR + PBS: $204.44 \pm 8.51$ versus AR + Dex: $86.15 \pm 7.65$ cardiomyocytes $\left./ \mathrm{mm}^{2} ; P<0.05\right)$ at $21 \mathrm{dpr}$ (Figure 3D). However, Dex treatment did not influence constitutive cardiomyocyte proliferation, suggesting that it inhibited only the injury-induced regenerative response, without any significant effects on normal cardiomyocyte homeostasis (Supplementary information, Figure S12). To further confirm the essential effects of the acute inflammatory response in reactive cardiomyocyte proliferation after AR in neonatal mice, the anti-Gr-1 (RB6-8C5) monoclonal antibody was used to deplete inflammatory leukocytes (neutrophils and some subsets of monocytes/macrophages [34, 35]), which were the main cellular constituents involved in cardiac acute inflammation. We found that cardiomyocyte proliferation was significantly decreased in the anti-Gr-1 (RB6-8C5) monoclonal antibody-treated group, which indicated that acute inflammation was indispensable for the injury-induced neonatal cardiomyocyte proliferation (Supplementary information, Figure S13). These results demonstrate that acute inflammatory response was necessary and sufficient for reactive cardiomyocyte proliferation in neonatal mouse hearts.

\section{IL-6 signaling is required for reactive cardiomyocyte proliferation in neonatal mice}

Our cytokine panel assays showed that IL-6 was the most upregulated cytokine after AR (Supplementary information, Figure S1). Previous studies have suggested that IL-6 signaling might play an essential role in tissue and organ regeneration [36, 37]. To investigate whether 
A

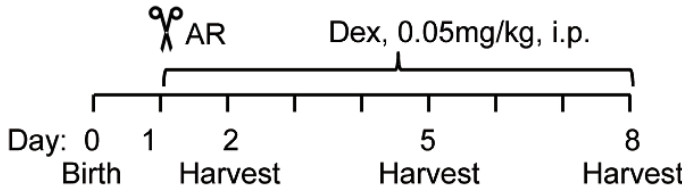

B

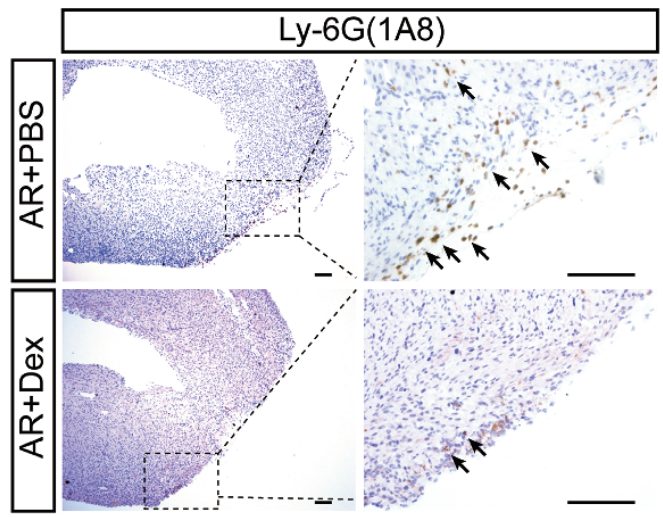

C

il6

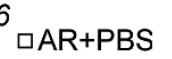

- $A R+D e x$

${ }^{i l 1 b}$ AR+PBS

- AR+Dex

$c c / 3 \square A R+P B S$

- $A R+D e x$

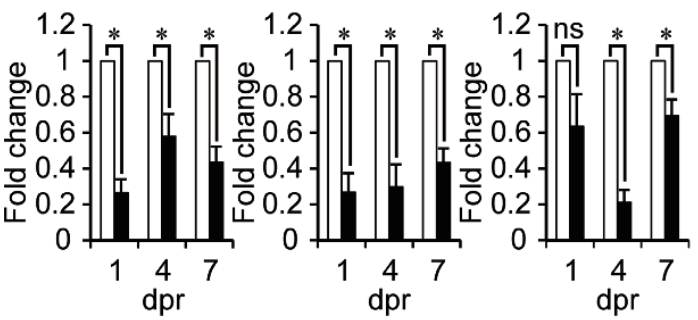

D

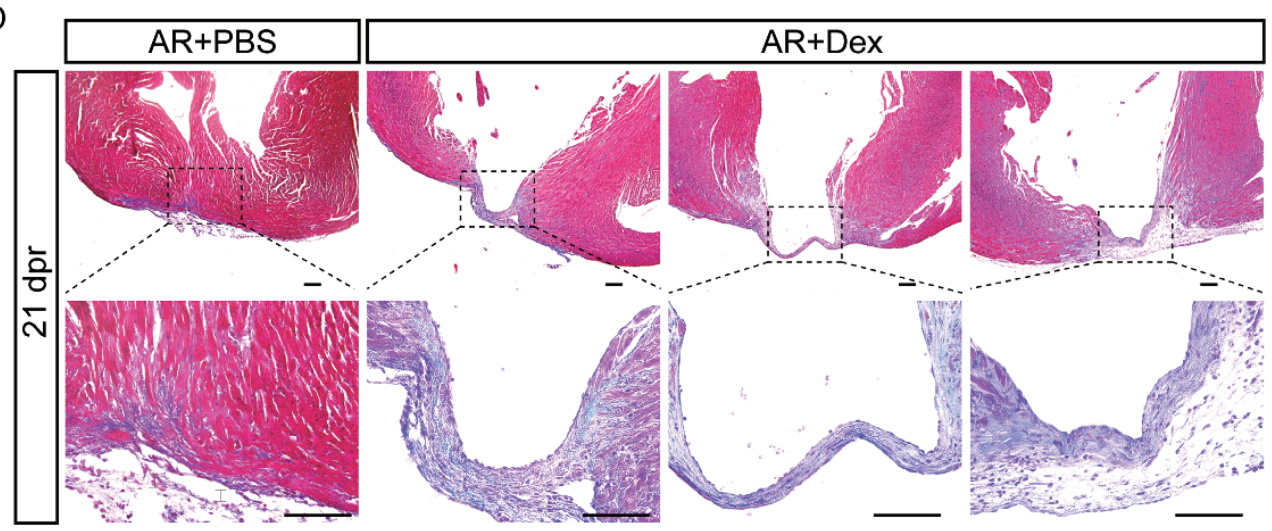

E

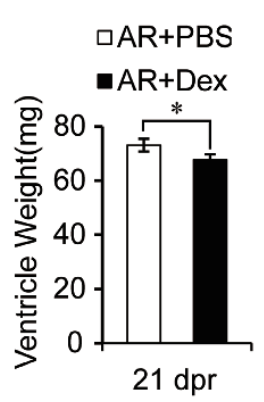

F

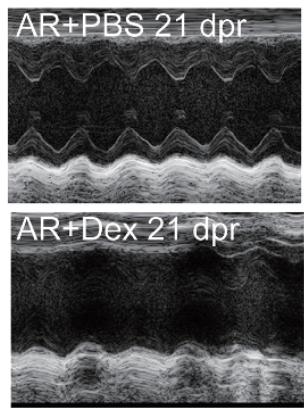

$\square \mathrm{AR}+\mathrm{PBS}$

$\square A R+P B S$

- $A R+D e x$

- $A R+D e x$
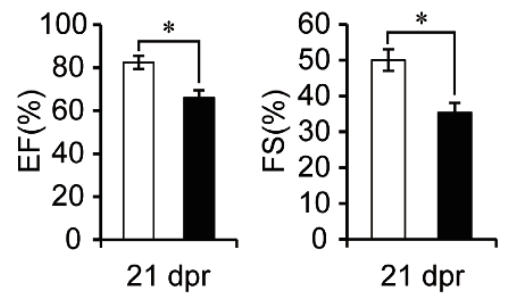

Figure 2 Injured hearts cannot regenerate after immunosuppression in neonatal mice. (A) Schematic showing dexamethasone (Dex) treatment after AR in neonatal 1-day-old mice. (B) Immunostaining for Ly-6G (1A8) in PBS- and Dex-treated hearts at $1 \mathrm{dpr}$. The high-magnification views of the boxed areas are presented on the right. Arrows indicate Ly- $6 \mathrm{G}(1 \mathrm{~A} 8)^{+}$ leukocytes. Scale bars, $100 \mu \mathrm{m}$. (C) qRT-PCR assays of the expression of $i / 6, i / 1 b$ and $c c / 3$. Gene expression data in the Dex-treated hearts are presented as a fold change compared to the PBS-treated hearts. $n=3$ per group. (D) Masson's staining of PBS- and Dex-treated hearts at $21 \mathrm{dpr}$. The high-magnification views of the boxed areas are presented below. $n=3$ per group; scale bars, $100 \mu \mathrm{m}$. (E) Quantification of ventricle weights of the PBS- and Dex-treated hearts at $21 \mathrm{dpr}$. $n=6$ per group. (F) Representative images of M-mode echocardiography of hearts at $21 \mathrm{dpr}$ between PBS- and Dex- treated mice. Systolic function of the left ventricular was measured by quantifying ejection fraction (EF) and fractional shortening (FS). $n=$ 3 per group. Values are presented as the mean $\pm S E M ; * P<0.05$; NS, not statistically significant. 
A

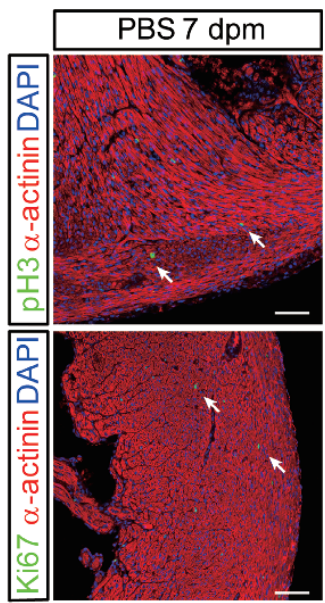

$\square P B S \backsim Z A$

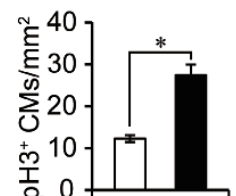

C

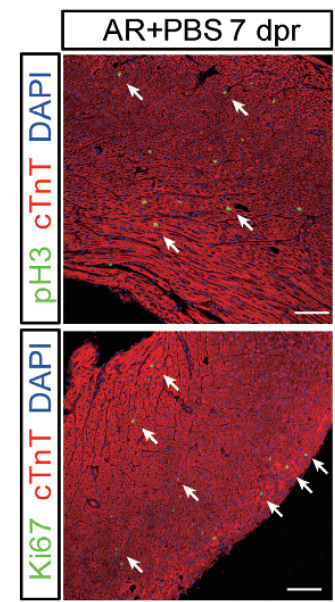

$\square A R+P B S$

- $A R+D e x$

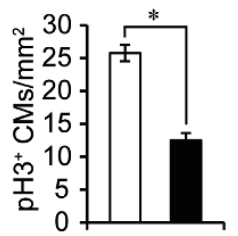

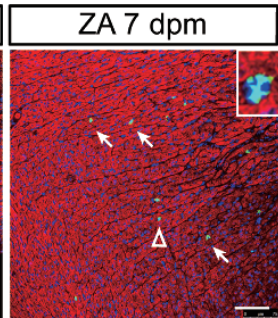

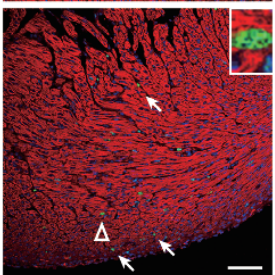

口PBS-ZA
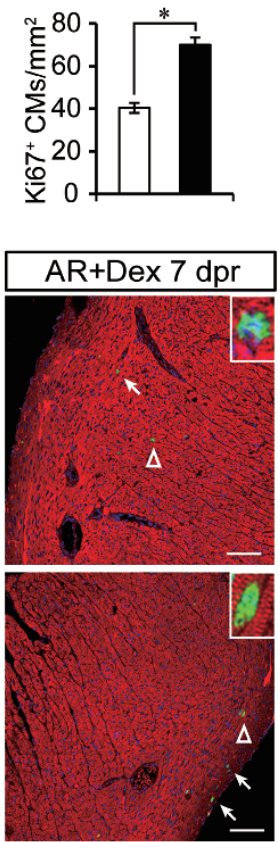

$\square A R+P B S$

- AR+Dex

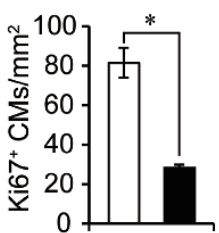

B

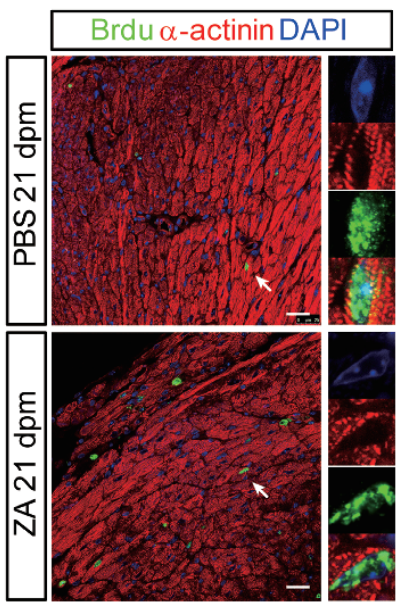

口PBS $=Z A$

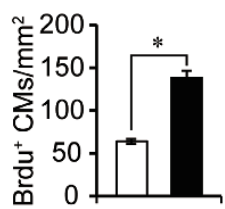

D

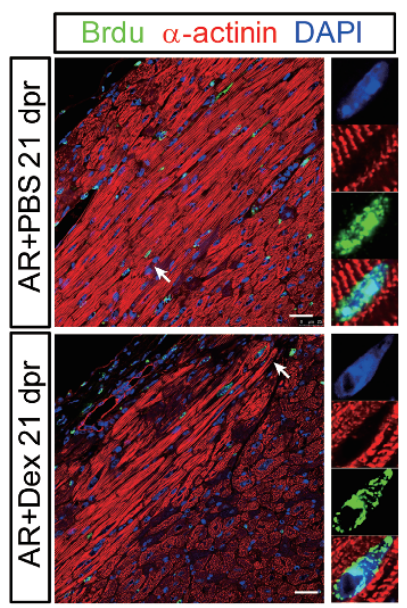

$\square A R+P B S$

- $A R+$ Dex

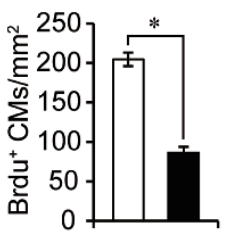

Figure 3 The acute inflammatory response is necessary and sufficient for reactive cardiomyocyte proliferation in neonatal mouse hearts. (A) Cardiomyocyte proliferation was identified by immunostaining for $\mathrm{pH} 3$ (green) and $\alpha$-actinin (red) co-localization and Ki67 (green) and $\alpha$-actinin (red) co-localization in PBS and ZA micro-injected hearts at $7 \mathrm{dpm}$. (B) The newly formed cardiomyocytes were shown as Brdu and a-actinin double-positive cells following a pulse-chase experiment in PBS and ZA micro-injected hearts at $21 \mathrm{dpm}$. (C) Immunostaining for pH3 (green) and Ki67 (green) with cardiac troponin T (cTnT) (red) co-localization in PBS- and Dex- treated hearts at $7 \mathrm{dpr}$. (D) The newly formed cardiomyocytes were shown as Brdu and $\alpha$-actinin double-positive cells in PBS- and Dex-treated hearts at $21 \mathrm{dpr}$. In $\mathbf{A}$ and $\mathbf{C}$, arrows indicate cardiomyocytes positive for $\mathrm{pH} 3$ or Ki67 staining. The inset is a high-magnification image of a pH3- or Ki67-positive cardiomyocyte which is denoted by an arrowhead. Scale bars, $75 \mu \mathrm{m}$. In $\mathbf{B}$ and $\mathbf{D}$, magnification images of a Brdu ${ }^{+}$cardiomyocyte (arrow) are shown on the right. Scale bars, $25 \mu \mathrm{m}$. CMs, cardiomyocytes; $n=3$ per group; values are presented as the mean \pm SEM; $* P<0.05$. 
IL-6 was necessary for the early cardiac regenerative response, we assessed reactive cardiomyocyte proliferation in IL-6 knockout (IL- $6^{-/}$) neonatal mouse hearts after AR. As shown in Supplementary information Figure S14, IL-6 expression was completely absent in IL- $6^{-/-}$neonatal mice, and cardiac injury failed to upregulate the expression of IL- 6 at 1 dpr in the IL- $6^{-/-}$hearts. IL- 6 deficiency also resulted in a significant decrease in $\mathrm{pH}^{+}$cardiomyocytes compared to wild-type (WT) hearts at $4 \mathrm{dpr}$ (WT: $30.5 \pm 1.83$ versus IL-6 $6^{--}: 17.56 \pm 1.87$ cardiomyocytes/ $\left.\mathrm{mm}^{2} ; P<0.05\right)$ and at $7 \mathrm{dpr}(\mathrm{WT}: 33.06 \pm 1.03$ versus IL$6^{-1}: 15.83 \pm 1.48$ cardiomyocytes $\left./ \mathrm{mm}^{2} ; P<0.05\right)$. Furthermore, a significant decrease in $\mathrm{Ki}^{+} 7^{+}$cardiomyocytes was observed in the IL- $6^{-/}$hearts at 4 dpr (WT: 67.37 \pm 2.85 versus IL- $6^{--}: 44.5 \pm 3.12$ cardiomyocytes $/ \mathrm{mm}^{2}$; $P<0.05)$ and at $7 \mathrm{dpr}\left(\mathrm{WT}: 68.83 \pm 2.64\right.$ versus IL-6 ${ }^{-1-}$ : $36.06 \pm 2.01$ cardiomyocytes $/ \mathrm{mm}^{2} ; P<0.05$; Figure 4A). The Brdu pulse-chase assay showed that deletion of IL-6 led to a significant decrease in $\mathrm{Brdu}^{+}$cardiomyocytes at $21 \mathrm{dpr}$ (WT: $213.4 \pm 14.05$ versus IL-6 ${ }^{-1}: 109.14 \pm 7.94$ cardiomyocytes $/ \mathrm{mm}^{2} ; P<0.05$; Figure 4B). In addition, neonatal IL- $6^{-/}$mice failed to regenerate the injured myocardium or restore normal systolic function at $21 \mathrm{dpr}$ compared with WT mice (Figure 4C and 4D).

Using apical intramyocardial microinjection of recombinant IL-6 protein in neonatal 1-day-old mice, we further explored whether IL-6 could induce cardiomyocyte proliferation without apparent cardiac lesions. The number of $\mathrm{pH}^{+}$cardiomyocytes in the IL- 6 micro-injected hearts substantially increased relative to the PBS micro-injected hearts at $4 \mathrm{dpm}$ (PBS: $13.85 \pm 0.73$ versus IL-6: $27.16 \pm 2.4$ cardiomyocytes $\left./ \mathrm{mm}^{2} ; P<0.05\right)$ and at 7 dpm (PBS: $15.78 \pm 1.45$ versus IL-6: $28.78 \pm 1.8$ cardiomyocytes $\left./ \mathrm{mm}^{2} ; P<0.05\right)$. Additionally, we observed a significant increase in $\mathrm{Ki} 67^{+}$cardiomyocytes in the IL-6 micro-injected hearts at $4 \mathrm{dpm}$ (PBS: $42.27 \pm 1.41$ versus IL-6: $58.08 \pm 1.93$ cardiomyocytes $/ \mathrm{mm}^{2} ; P<0.05$ ) and at $7 \mathrm{dpm}$ (PBS: $37.6 \pm 2.85$ versus IL-6: $60.45 \pm$ 2.61 cardiomyocytes $/ \mathrm{mm}^{2} ; P<0.05$; Figure 4E). We also found an increase in $\mathrm{Brdu}^{+}$cardiomyocytes in the IL-6 micro-injected hearts (PBS: $63.53 \pm 6.68$ versus IL-6: $142.71 \pm 7.67$ cardiomyocytes $\left./ \mathrm{mm}^{2} ; P<0.05\right)$ at $21 \mathrm{dpm}$ (Figure 4F). These results indicate that IL-6 signaling is required for reactive cardiomyocyte proliferation and the cardiac regenerative process in neonatal mouse hearts.

STAT3 plays a key role in reactive cardiomyocyte proliferation in the neonatal mouse heart

To further examine how IL- 6 mediates reactive cardiomyocyte proliferation, we assessed three major downstream intracellular signaling pathways, MEK/ERK, JAK/STAT3 and PI3K/AKT, by western blotting. After
AR, only JAK/STAT3 signaling was substantially activated, as shown by a clear increase in phospho-STAT3 (p-STAT3) (Tyr705), which was confirmed in resected hearts, ZA micro-injected hearts and IL-6 micro-injected hearts via p-STAT3 (Tyr705) immunostaining (Figure 5A and $5 \mathrm{~B})$. To investigate whether STAT3 signaling was required for reactive cardiomyocyte proliferation, we performed AR in cardiomyocyte-specific STAT3 conditional knockout neonatal 1-day-old mice and then evaluated cardiomyocyte proliferation. To test the efficiency of gene deletion, we examined the expression of STAT3 in the hearts of $\alpha \mathrm{MHC}-\mathrm{MerCreMer}$; STAT3 $3^{\mathrm{t} / \mathrm{fl}}$ mice after tamoxifen treatment. As expected, STAT3 expression was efficiently ablated in the hearts of aMHC-MerCreMer; $\mathrm{STAT}^{\mathrm{f} / \mathrm{fl}}$ mice after tamoxifen induction (Supplementary information, Figure S15). STAT3 deficiency $\left(\mathrm{STAT}^{-/-}\right.$) led to a significant decrease in $\mathrm{pH}^{+}$cardiomyocytes at 4 dpr $\left(\right.$ STAT3 $^{+/+}: 30.27 \pm 1.92$ versus STAT3 ${ }^{-/}: 18.32 \pm 1.91$ cardiomyocytes $\left./ \mathrm{mm}^{2} ; P<0.05\right)$ and at $7 \mathrm{dpr}\left(\mathrm{STAT}^{+++}\right.$: $31.76 \pm 3.01$ versus STAT3 ${ }^{-1}: 19.14 \pm 1.87$ cardiomyocytes $\left./ \mathrm{mm}^{2} ; P<0.05\right)$. In the STAT3 ${ }^{-/-}$hearts, there was also a decreased number of $\mathrm{Ki} 7^{+}$cardiomyocytes relative to the $\mathrm{STAT3}^{+/+}$hearts at $4 \mathrm{dpr}\left(\mathrm{STAT}^{+/+}: 61.04\right.$ \pm 2.78 versus $\mathrm{STAT3}^{-1}: 38.07 \pm 2.71$ cardiomyocytes/ $\left.\mathrm{mm}^{2} ; P<0.05\right)$ and $7 \mathrm{dpr}\left(\mathrm{STAT3}^{+/+}: 63.24 \pm 2.52\right.$ versus STAT3 $^{-/}: 33.56 \pm 1.52$ cardiomyocytes $\left./ \mathrm{mm}^{2} ; P<0.05\right)$ (Figure 5C). The number of $\mathrm{Brdu}^{+}$cardiomyocytes in the STAT3 ${ }^{-1-}$ hearts was significantly reduced relative to the STAT3 ${ }^{+/+}$hearts $\left(\right.$STAT3 $^{+/+}: 207.19 \pm 11.15$ versus STAT3 $^{-1-}: 104.39 \pm 6.17$ cardiomyocytes $\left./ \mathrm{mm}^{2} ; P<0.05\right)$ at $21 \mathrm{dpr}$ (Figure 5D). Additionally, IL-6 was found to be sufficient to induce neonatal cardiomyocyte proliferation in vitro, which could be blocked by the STAT3 inhibitor, S3I-201 (Figure 6). These data indicate that activation of STAT3 downstream of IL-6 is required for reactive cardiomyocyte proliferation after cardiac AR in neonatal mice.

\section{Discussion}

After heart damage, acute inflammation is one of the first responses detected in the injured myocardium in both neonates and adults. Myeloid cells, including neutrophils and monocytes/macrophages, are rapidly recruited to the injured tissue, and various cytokines are released to regulate the microenvironment of the injured myocardium [38]. Cardiac inflammation is not capable of stimulating a significant regenerative response in adult mouse hearts, but instead triggers a robust fibrotic repair response [8, 23-25]; however, in the context of cardiac AR in neonatal 1-day-old mice, the robust inflammatory response did not block heart regeneration [8]. Further- 
A

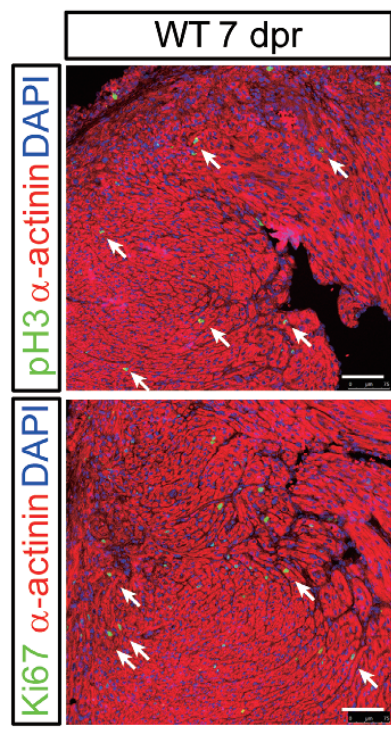

口WT $=\mathrm{IL}-6^{-/ 2}$

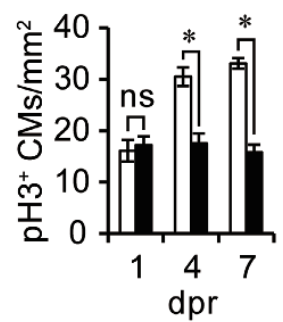

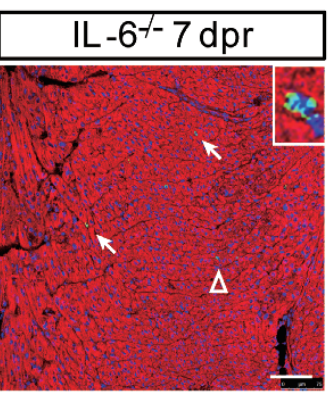

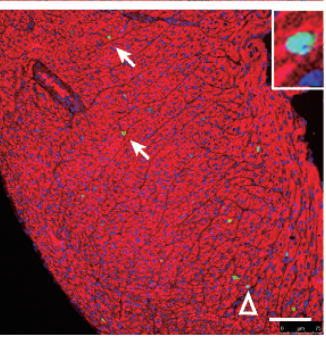

口WT $\square \mathrm{IL}-6^{-1-}$

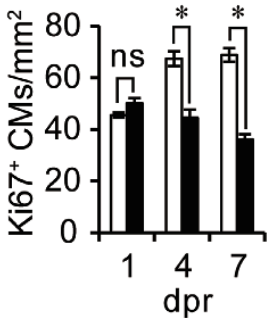

B

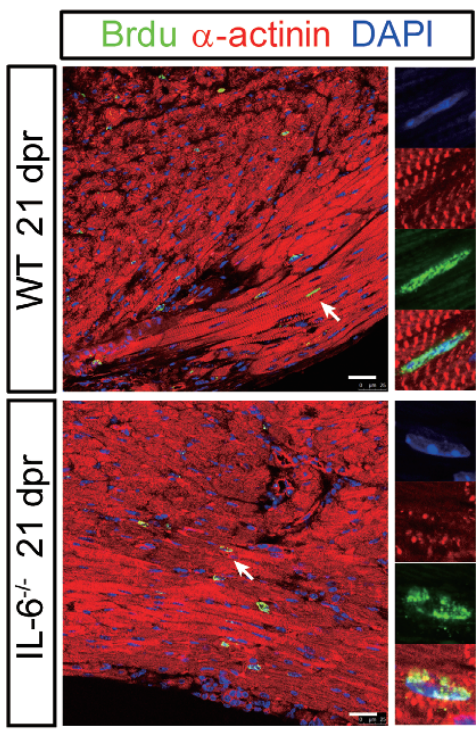

口WT $\square \mathrm{LL}-6^{-1-}$

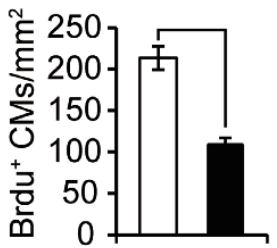

C

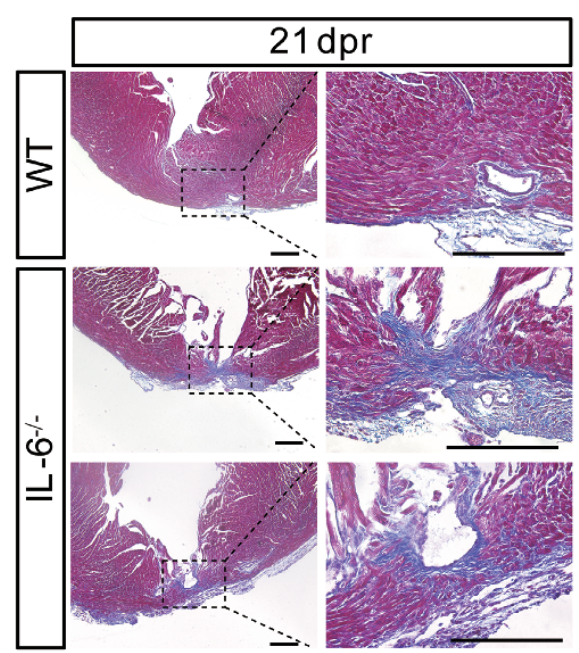

E

口PBS $\square \mathrm{IL}-6$

口PBS $=I L-6$
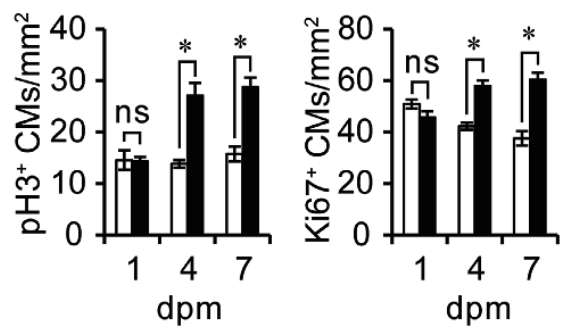

D
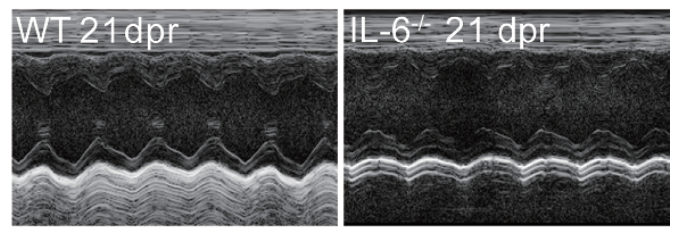

$\square \mathrm{WT} \backsim \mathrm{IL}-6^{-/}$

口WT $\square \mathrm{LL}-6^{-/}$
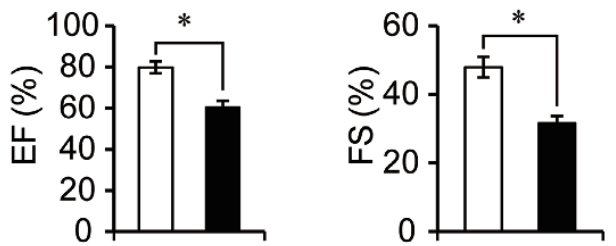

F $\quad$ 口PBS $\square \mathrm{IL}-6$

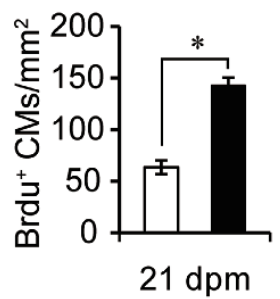


more, neonatal mouse heart regeneration appeared to require an initial inflammatory response after myocardial injury. This inflammatory response after myocardial damage differs qualitatively and quantitatively between the 1-day-old (regenerative) and 14-day-old (non-regenerative) mice. Neonatal cardiac macrophages are required for replacing the injured myocardium following MI $[7,21]$. Here, we found that an apical intramyocardial microinjection of ZA could induce an acute inflammatory response without any apparent cardiac lesions in neonatal mice. Moreover, the triggered inflammation increased cardiomyocyte proliferation, which is considered to be an efficient regenerative response once neonatal mouse hearts are injured. In contrast, cardiomyocyte proliferation after AR was inhibited in immunosuppressed neonatal mice. Our results showed that acute inflammation was necessary and sufficient for stimulating the cardiac regenerative response in neonatal 1-day-old mice.

Furthermore, we found that IL-6 signaling, as a major component of inflammation, was able to induce cardiomyocyte proliferation in neonatal mice. This result is in agreement with the results of a previous report, which indicated that recombinant IL-6 treatment stimulated neonatal cardiomyocyte proliferation in vitro [39]. As a pro-inflammatory cytokine, IL-6 binds to the IL-6 receptor and the glycoprotein 130 complex receptor and then activates STAT3 through tyrosine phosphorylation [40]. Our findings revealed that STAT3, as the downstream mediator of IL-6 signaling, was required for reactive cardiomyocyte proliferation induced by myocardial injury in neonatal mice. A previous profiling study also identified that cardiomyocyte STAT3 activation was required for injury-induced cardiomyocyte proliferation and heart regeneration in adult zebrafish [41]. Additionally, IL-6STAT3 signaling is required for muscle regeneration [36, 42]. IL-6, which is derived from infiltrating macrophages [42], fibro/adipogenic progenitors [43] and satellite cells $[44,45]$, can facilitate muscle regeneration by promoting satellite cell proliferation and myogenic differentiation.
IL-6-STAT3 signaling also plays an essential role in normal liver regeneration after hepatic injury [37, 46-48]. In particular, IL-6 produced by hepatic Kupffer cells (liver macrophages) contributes to hepatocyte proliferation during the process of liver regeneration. Furthermore, STAT3 fosters hepatocyte proliferation and the IL-6-mediated hepatic growth response during liver regeneration.

According to recent reports, the regenerative and repair capacity of neonatal hearts varies with different types of injury [8, 18, 49-54]. Porrello et al. [8] found that the hearts of 1-day-old neonatal mice could regenerate completely after AR (approximately $15 \%$ of the ventricular myocardium) over a 21-day period, which was confirmed in our research and other studies [51, 55-57]. The conflicting results reported by Andersen et al. [49] and Bryant et al. [50] were mainly due to the resection of much more myocardium. Furthermore, Porrello et al. [18] and Haubner et al. [58] showed that the hearts of neonatal mice could also regenerate after MI. Nevertheless, Konfino et al. [51] claimed that neonatal MI leads to an incomplete regeneration with a small, thin scar at the injured area. The difficulties in standardizing the operation of MI in neonatal mice may account for the discrepancy in the reported regenerative potential [51]. On the contrary, Darehzereshki et al. [52] and Polizzotti et al. [53] discovered that the hearts could not regenerate after cryoinjury in neonatal mice. We speculate that the differences in the extent of injury and the microenvironment at the injury site between cryoinjury and AR might have led to these different types of myocardial repair responses. Thus, the modes and extent of cardiac injury may dictate the course of regenerative and repair responses. As to our experiments, we strictly followed the original protocol for all of the neonatal AR procedures [8].

In summary, our results provide evidence that acute cardiac inflammation is required and sufficient for stimulating cardiomyocyte proliferation in neonatal mice. In particular, IL-6-STAT3 signaling is responsible for reactive cardiomyocyte proliferation after cardiac injury

Figure 4 IL-6 signaling is required for the cardiac regenerative response in neonatal mice. (A) Cardiomyocyte proliferation was identified by immunostaining for $\mathrm{pH} 3$ (green) and Ki67 (green) together with $\alpha$-actinin (red) co-localization in wild-type (WT) and IL-6 knockout (IL-6 $6^{-/}$) hearts at 7 dpr in neonatal mice. Arrows indicate cardiomyocytes positive for pH3 or Ki67 staining. The inset is a high-magnification image of a pH3- or Ki67-positive cardiomyocyte which is denoted by an arrowhead. Scale bars, $75 \mu \mathrm{m}$. (B) The newly formed cardiomyocytes were shown as Brdu and $\alpha$-actinin double-positive cells in WT and $\mathrm{IL}-6^{-/-}$hearts at $21 \mathrm{dpr}$. Magnification images of the Brdu ${ }^{+}$cardiomyocyte (arrow) are shown on the right. Scale bars, $25 \mu \mathrm{m}$. (C) Masson staining of WT and IL- $6^{-/-}$hearts at $21 \mathrm{dpr}$. The high-magnification views of the boxed areas are presented on the right. Scale bars, $200 \mu \mathrm{m}$. (D) Representative images of M-mode echocardiography of hearts at $21 \mathrm{dpr}$ between WT and IL-6 ${ }^{-/-}$mice. Left ventricular systolic function was quantified by EF and FS. (E) Quantification of cardiomyocyte proliferation in the PBS and IL-6 micro-injected hearts at 1, 4 and $7 \mathrm{dpm}$ in neonatal mice. (F) Quantification of newly formed cardiomyocytes in the PBS and IL-6 micro-injected hearts at $21 \mathrm{dpm}$. CMs, cardiomyocytes; $n=3$ per group; values are presented as the mean $\pm \mathrm{SEM} ;{ }^{*} P<0.05$; NS, not statistically significant. 


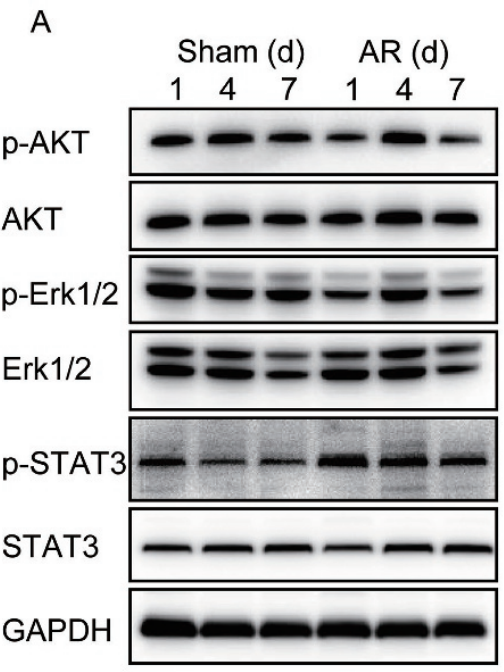

B

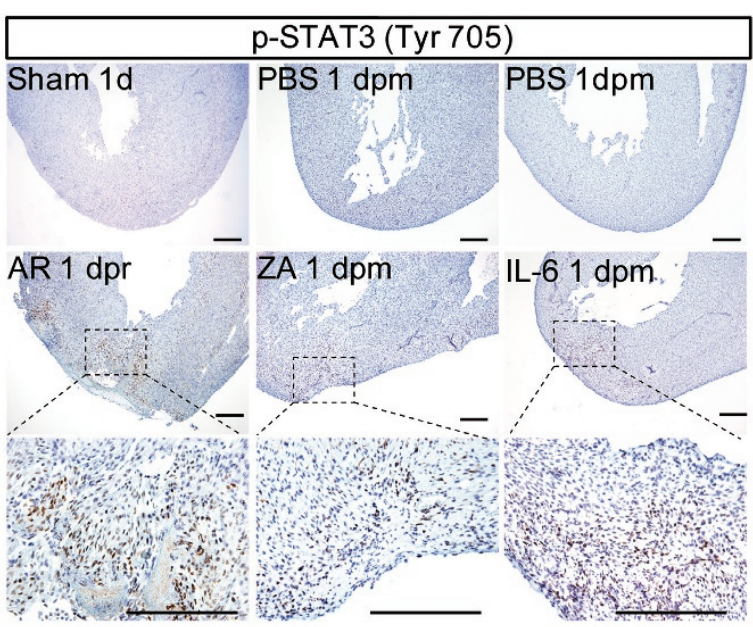

C
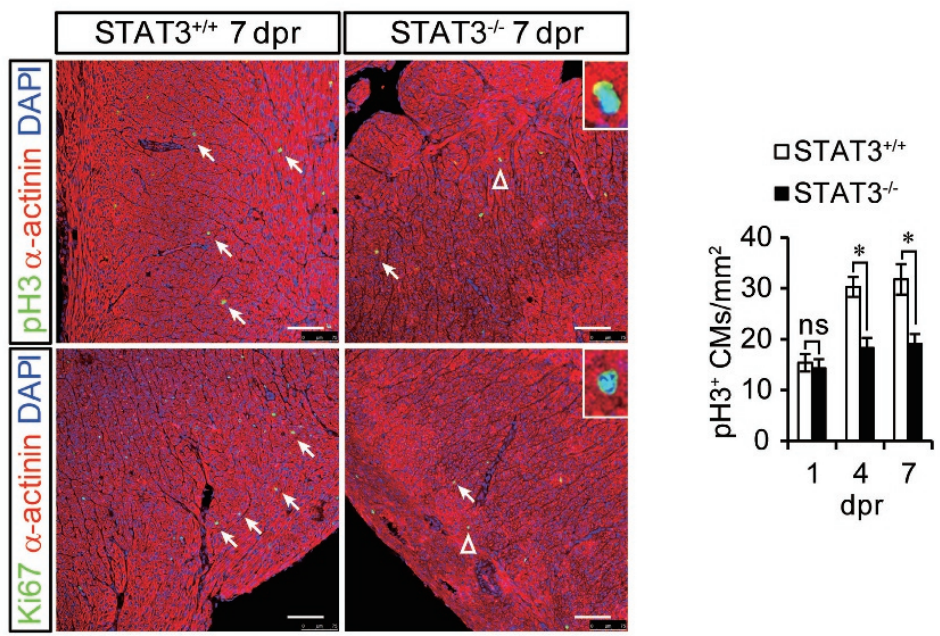

$$
\begin{aligned}
& \text { 口STAT3 }{ }^{+/+} \\
& \text {-STAT } 3^{--}
\end{aligned}
$$

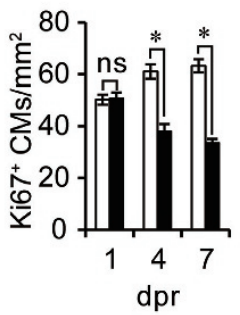

D
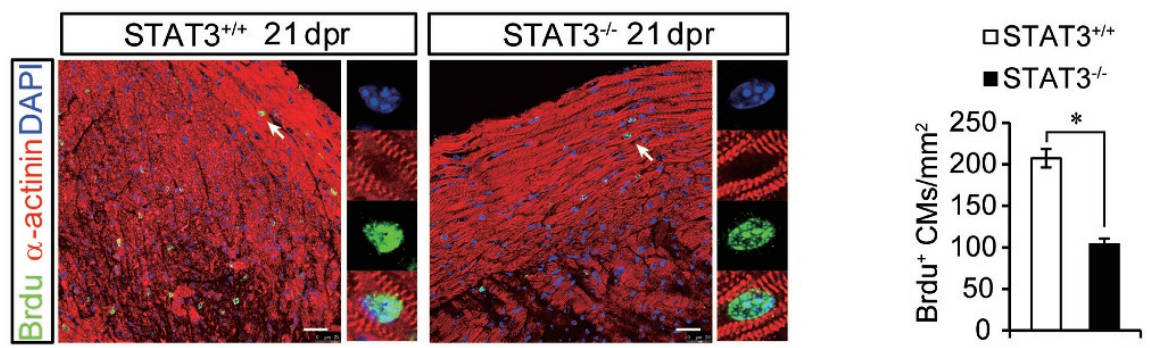

Figure 5 Stat3 plays a key role in the regenerative response in the neonatal mouse heart. (A) Western blotting analyses of phospho-AKT and AKT, phospho-Erk1/2 and Erk1/2, and phospho-STAT3 and STAT3 between the sham and AR hearts at 1, 4 and 7 dpr. (B) Immunostaining for phospho-STAT3 (Tyr705) in the resected, ZA micro-injected and IL-6 micro-injected hearts at 1 day post-surgery in neonatal mouse hearts. The high-magnification views of the boxed areas are presented below. Scale bars, $200 \mu \mathrm{m}$. (C) Cardiomyocyte proliferation was identified by immunostaining for pH3 (green) and Ki67 (green) together with $\alpha$-actinin (red) co-localization in the STAT3 ${ }^{+/+}$and STAT3 ${ }^{-/-}$mice at $7 \mathrm{dpr}$ in neonatal mice. Arrows indicate cardiomyocytes positive for $\mathrm{pH} 3$ or Ki67 staining. The inset is a high-magnification image of a pH3- or Ki67-positive cardiomyocyte which is denoted by an arrowhead. Scale bars, $75 \mu \mathrm{m}$. (D) The newly formed cardiomyocytes were shown as Brdu and $\alpha$-actinin double-positive cells following a pulse-chase experiment in STAT3 ${ }^{+/}$and STAT3 ${ }^{-/-}$hearts at $21 \mathrm{dpr}$. Magnification images of the $\mathrm{Brdu}^{+}$cardiomyocyte (arrow) are shown on the right. Scale bars, $25 \mu \mathrm{m}$. CMs, cardiomyocytes; $n=3$ per group; values are presented as the mean $\pm \mathrm{SEM} ; * P<0.05$. 
of neonatal mice. These findings suggest that modulation of the acute inflammatory response, particularly IL-6STAT3 signaling, may have important implications in regenerative medicine for the treatment of human cardiac diseases.

\section{Materials and Methods}

\section{Mice}

Neonatal 1-day-old mice of both sexes were used in our study. The ICR/CD-1 and C57BL/6J WT mice were acquired from Vital River Laboratory Animal Technology Co. Ltd. (Beijing, China) The IL-6 $6^{-/}$(B6.129S2-I16tm1Kopf/J) [59], STAT3 ${ }^{\mathrm{f} /}$ ${ }^{\mathrm{fl}}$ (B6.129S1-Stat3tm1Xyfu/J) [60] and $\alpha$ MHC-MerCreMer mice (B6.FVB(129)-Tg(Myh6-cre/Esr1*)1Jmk/J) [61] were acquired from the Jackson Laboratory, Bar Harbor, ME. Tamoxifen (Sigma) was administered to the $\alpha \mathrm{MHC}-\mathrm{MerCreMer}$; STAT $3^{\mathrm{t} / \mathrm{fl}}$ mice $\left(\mathrm{STAT}^{-/-}\right.$mice) and $\mathrm{STAT}^{\mathrm{H} / \mathrm{fl}}$; $\mathrm{STAT}^{\mathrm{H} / \mathrm{fl}}$ mice $\left(\mathrm{STAT3}^{+/+}\right.$mice) at birth (postnatal day 0), according to a protocol published previously [62]. On the next day of tamoxifen injection, the neonatal mice underwent AR. All of the animal procedures were approved by the Care of Experimental Animals Committee of the Chinese Academy of Medical Sciences and Peking Union Medical College, China.

\section{Apical resection}

AR was performed on neonatal 1-day-old mice (ICR/CD-1 strain, Vital River Laboratory Animal Technology Co., Ltd., China) as previously described $[8,57]$. Briefly, neonatal 1-day-old mice were anesthetized by hypothermia on an ice bed for $4 \mathrm{~min}$. Left parasternal thoracotomy at the fourth intercostal space was performed by blunt dissection following skin incision. The cardiac apex was resected using a pair of iridectomy scissors. AR was successfully achieved while the left ventricle chamber was exposed. Then, the resected mice were immediately removed from the ice beds, and the thoracic wall and skin incisions were sutured with 8-0 non-absorbable silk suture. The neonatal mice were warmed for several minutes under a heat lamp until recovery. The same procedures were performed without AR in the sham-operated groups.

\section{Dexamethasone treatment}

Dexamethasone (Dex, $0.05 \mathrm{mg} / \mathrm{kg}$, Sigma) was administered daily from the day of AR in the 1-day-old neonates to $7 \mathrm{dpr}$ by i.p. injection. The control mice were treated with PBS (same volume as that of Dex). To examine whether the Dex treatment significantly inhibited heart regeneration, we weighed the ventricles of the PBS- and Dex-treated hearts at $21 \mathrm{dpr}$, as described previously [8].

\section{Anti-Gr-1 antibody treatment}

We used anti-Gr-1(RB6-8C5) antibody (Bio X Cell) treatment to deplete inflammatory cells (neutrophils and some subsets of monocytes/macrophages) [35, 63]. Anti-Gr-1(RB6-8C5) antibody $(20 \mu \mathrm{g} / \mathrm{g})$ was administered i.p. immediately at birth (postnatal day 0 ) and $12 \mathrm{~h}$ after (postnatal day 0.5). On the following day (postnatal day 1), the neonatal mice underwent AR and received antiGr-1(RB6-8C5) antibody treatment after recovery and again $12 \mathrm{~h}$ later. Then, anti-Gr-1(RB6-8C5) antibody was administered daily until the heart samples were harvested. The control mice were treated with the IgG isotype antibody.

\section{Anti-IL-6 antibody treatment}

Anti-IL-6 antibody $(30 \mu \mathrm{g} / \mathrm{g})$ was administered i.p. to block IL-6 activity immediately at birth (postnatal day 0 ). Twelve hours later (postnatal day 0.5), the neonatal mice underwent AR and received anti-IL-6 antibody treatment after recovery and again $12 \mathrm{~h}$ later. Then, anti-IL-6 antibody was administered daily until heart samples were harvested. The control mice were treated with $\operatorname{IgG}$ isotype control antibody.

\section{Apical intramyocardial microinjection}

Neonates (ICR/CD-1 strain, Vital River Laboratory Animal Technology Co., Ltd., China) were anesthetized by cooling on an ice bed for $4 \mathrm{~min}$, as described previously [8]. Lateral thoracotomy via the fourth left-intercostal space was performed by blunt dissection of the intercostal muscles following skin incision. The chest was opened, and $5 \mu \mathrm{g}$ of ZA (S. cerevisiae) BioParticles, Alexa Fluor 488 Conjugate (Invitrogen), or ZA (S. cerevisiae) BioParticles, unlabeled (Invitrogen), in $0.5 \mu 1$ of sterile PBS solution was micro-injected intramyocardially into the apical myocardium through a 36-gauge needle (World Precision Instruments Inc., Sarasota, FL, USA). Successful microinjection was indicated by the presence of Alexa Fluor ${ }^{\circledR} 488$ at the site of microinjection. Control neonatal 1-day-old mice were micro-injected with $0.5 \mu 1$ of a sterile PBS solution. Recombinant murine IL-6 (IL-6, Peprotech, $10 \mathrm{ng}$ in $0.5 \mu 1$ sterile PBS) was micro-injected according to the protocol described above. The control mice were micro-injected with $0.5 \mu 1$ of a sterile PBS solution.

\section{Neonatal rat cardiomyocyte isolation and culture}

Neonatal 1-day-old rat cardiomyocytes were prepared as previously described [64], with minor modifications. Briefly, neonatal rat cardiomyocytes were isolated by enzymatic disassociation of 1-day-old neonatal hearts. Cardiomyocytes were plated separately for $2 \mathrm{~h}$ to remove fibroblasts. The cells were resuspended in Dulbecco's modified Eagle's medium containing $4.5 \mathrm{~g} / \mathrm{l}$ glucose (DMEM, Life Technologies) and supplemented with 5\% FBS, $20 \mu \mathrm{g} / \mathrm{ml}$ vitamin B12, $100 \mathrm{U} / \mathrm{ml}$ of penicillin and $100 \mu \mathrm{g} / \mathrm{ml}$ of streptomycin. After $24 \mathrm{~h}$ of plating, the cells were incubated in serum-free medium overnight. For the western blot analysis, the cardiomyocytes were treated with $20 \mathrm{ng} / \mathrm{ml}$ IL-6 (Peprotech, Rocky Hill, NJ) or DMSO (Sigma) as a control for 15 min. The cardiomyocytes were pretreated with $100 \mu \mathrm{M} \mathrm{S} 3 \mathrm{I}-201$ (STAT3 inhibitor) for $2 \mathrm{~h}$ and treated with IL-6 and S3I-201 (Merck Millipore) for 15 $\min$. For the immunofluorescence assay, the cardiomyocytes were treated with $20 \mathrm{ng} / \mathrm{ml}$ IL-6 (Peprotech, Rocky Hill, NJ) or DMSO as a control for $24 \mathrm{~h}$. The cardiomyocytes were pretreated with $100 \mu \mathrm{M} \mathrm{S} 3 \mathrm{I}-201$ for $2 \mathrm{~h}$ and treated with IL-6 and S3I-201 for $24 \mathrm{~h}$.

\section{In vivo Brdu pulse-chase labeling experiments}

$35 \mu 1$ of Brdu $(20 \mathrm{mg} / \mathrm{ml})$ (Sigma) in PBS was administered subcutaneously to the neonatal mice at 1,7 and $14 \mathrm{dpr}$ or $\mathrm{dpm}$. At $21 \mathrm{dpr}$ or dpm, the hearts were harvested, and Brdu staining was performed as described previously [8].

\section{Quantitative reverse transcriptase-polymerase chain reac-} tion 
A

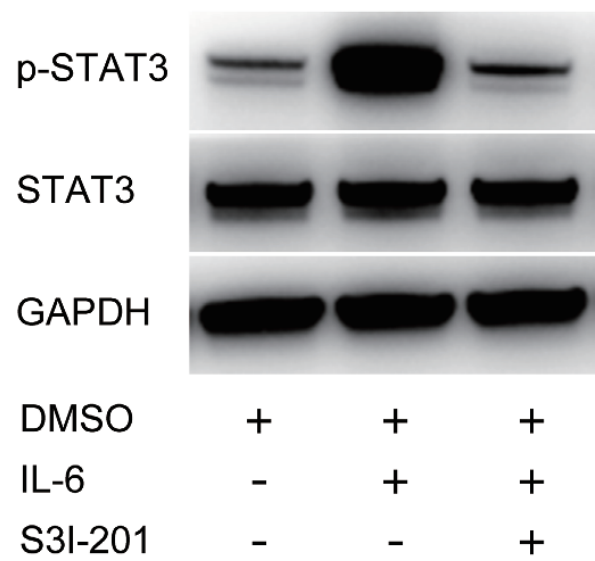

B
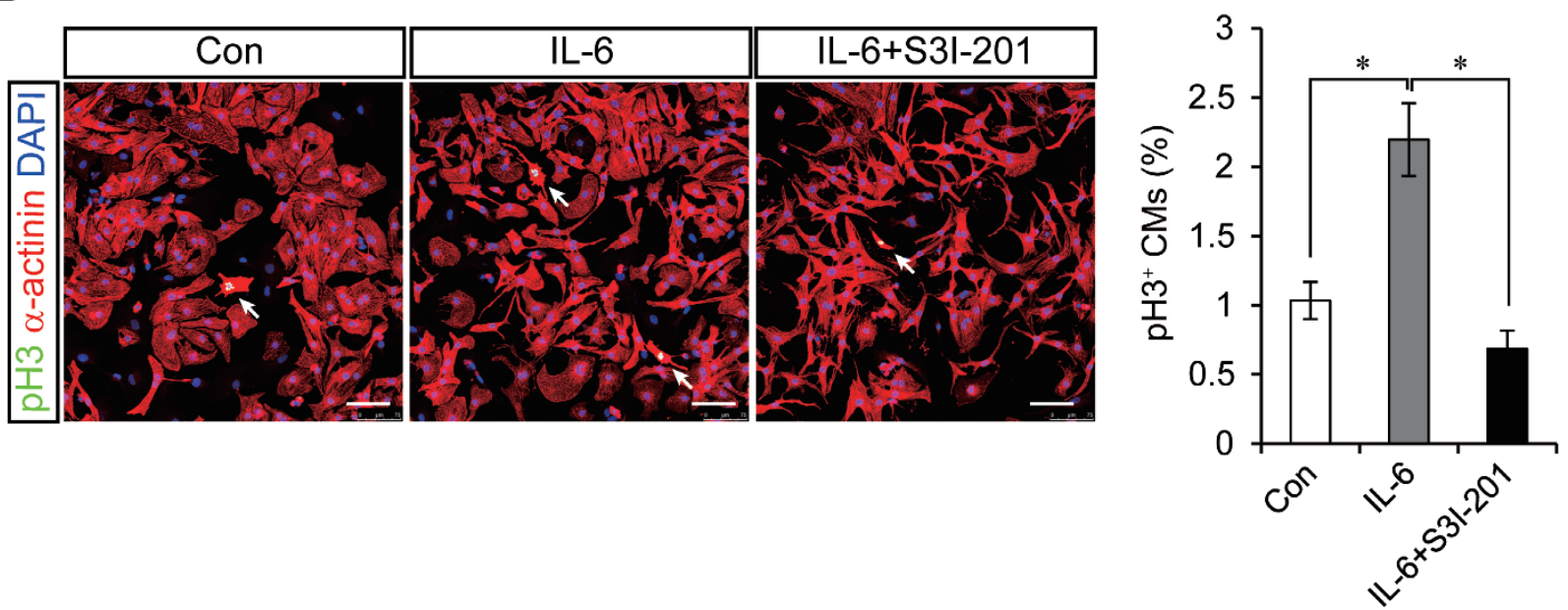

C
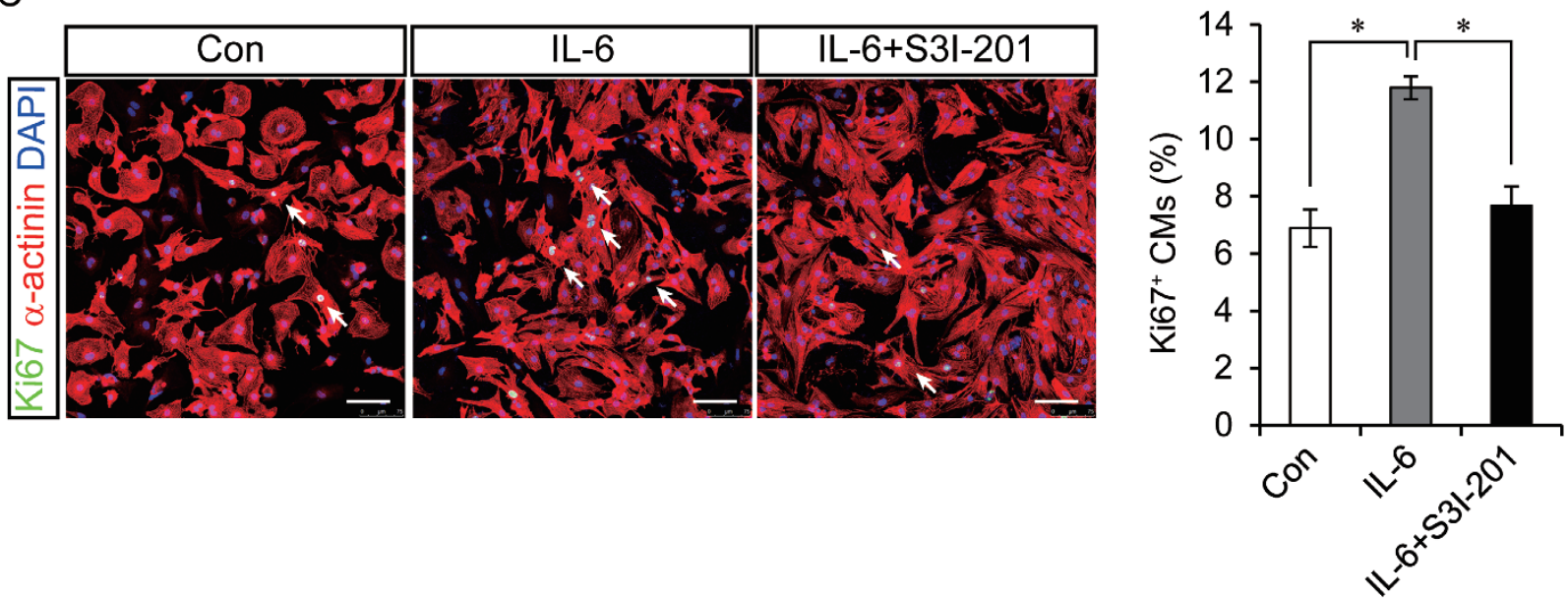

Figure 6 The pro-proliferative activity of IL-6 on neonatal cardiomyocytes is mediated by cardiomyocyte STAT3 signaling. (A) Western blotting analyses of phospho-STAT3 (Tyr705) (p-STAT3) and STAT3 after in vitro treatment of neonatal rat ventricular cardiomyocytes with DMSO, IL-6 or both IL-6 and S3I-201 (STAT3 inhibitor). (B, C) Immunofluorescence analysis of pH3 (green) and a-actinin (red) co-localization, and Ki67 (green) and a-actinin (red) co-localization in response to IL-6 or both IL-6 and S3I-201 administration in vitro in neonatal ventricular cardiomyocytes of rats. CMs, cardiomyocytes. Scale bars, $75 \mu \mathrm{m}$. Values are presented as the mean $\pm \mathrm{SEM} ;{ }^{*} P<0.05$. 
One-third of the ventricular myocardium containing the resection plane was dissected to enrich for transcripts. RNA was extracted from the myocardium using Trizol reagent (Invitrogen). cDNA was synthesized from $1 \mu \mathrm{g}$ of RNA using Oligo dT and MMLV reverse transcriptase (Invitrogen). Quantitative reverse transcriptase-PCR was performed with the SYBR Green detection system. All of the PCR experiments were performed with the Applied Biosystems 7300 Real Time PCR System. The transcript quantities were compared using the $\triangle \mathrm{CT}$ method. GAPDH was used as a housekeeping control gene. All of the primers for the SYBR green reactions are listed below [8].

il6 forward: 5'-TGATGCACTTGCAGAAAACA-3'

il6 reverse: 5'-ACCAGAGGAAATTTTCAATAGGC-3'

$i l 1 b$ forward: 5'-TCCAGGATGAGGACATGAGCAC-3'

$i l 1 b$ reverse: 5'-GAACGTCACACACCAGCAGGTTA-3'

$c c l 3$ forward: 5'-CATGACACTCTGCAACCAAGTCTTC-3'

ccl3 reverse: 5'-GAGCAAAGGCTGCTGGTTTCA-3'

STAT3 forward: 5'-TGCACCTGATCACCTTCGAGAC-3'

STAT3 reverse: 5'-CCCAAGCATTTGGCATCTGAC-3' gapdh forward: 5'-AAATGGTGAAGGTCGGTGTGAAC-3' gapdh reverse: 5'-CAACAATCTCCACTTTGCCACTG-3'

\section{Histology}

The harvested hearts were fixed in 4\% paraformaldehyde (PFA) at room temperature for $24 \mathrm{~h}$ and then were dehydrated in an ethanol and xylene series followed by paraffin embedding. The sectioned tissues ( $3 \mu \mathrm{m}$ thick) were cut through the entire ventricle, deparaffinized in xylene and rehydrated in ethanol. Hematoxylin and eosin and Masson's trichrome staining were performed according to standard procedures.

\section{Immunohistochemistry}

The hearts were fixed overnight with 4\% PFA and embedded in paraffin. Ly-6G (1A8) (BD Pharmingen), phospho-STAT3 (Tyr705) (Cell Signaling Technology) or endomucin (R\&D systems) immunostaining was conducted on $3-\mu \mathrm{m}$ tissue sections using the Streptavidin-Peroxidase Kit (Zhongshan jinqiao, Beijing, China), followed by staining with hematoxylin. The slides were dehydrated in ethanol twice for 3 min each and were subsequently mounted for light microscopy.

\section{Immunofluorescence}

For immunofluorescence staining, the paraffin-embedded sections were deparaffinized in xylene and rehydrated through graded ethanol. Heat-mediated antigen retrieval with an EDTA solution was performed to expose the antigen epitopes. The sections were permeabilized with $0.3 \%$ Triton X-100 in PBS, blocked with $10 \%$ goat serum (Invitrogen) and incubated with primary antibody overnight at $4{ }^{\circ} \mathrm{C}$. On the following day, the slides were washed three times in PBS and were then incubated with the secondary antibody for $45 \mathrm{~min}$ at $37{ }^{\circ} \mathrm{C}$. The slides were again washed three times in PBS in a dark room, stained with DAPI (Sigma) for 15 min to label the nuclei and mounted in Vectashield (Vector Labs, CA, USA). The primary antibodies included anti-cTnT (mouse monoclonal, Abcam), anti-pH3 (rabbit monoclonal, Abcam), anti-Ki67 (rabbit monoclonal, Abcam), anti-Brdu (mouse monoclonal, Cell Signaling Technology), anti- $\alpha$-actinin (mouse monoclonal, Sigma), anti- $\alpha$-actinin (rabbit monoclonal, Cell Signaling Technology) and anti-PECAM antibody (rabbit polyclonal, Abcam). The sec- ondary antibodies were conjugated to Alexa Fluor 488 (donkey anti-mouse, Invitrogen), Alexa Fluor 555 (donkey anti-rabbit, Invitrogen), and Alexa Fluor 633 (donkey anti-mouse, Invitrogen). Fluorescence was observed under a Leica SP8 confocal laser scanning microscope.

Immunofluorescence staining was also performed on cultured neonatal rat cardiomyocytes. For cardiomyocyte staining, the cells were fixed with $4 \%$ PFA and then permeabilized with $0.3 \%$ Triton/ PBS. The cells were blocked in $1 \%$ bovine serum albumin (BSA) and then incubated with the indicated antibodies. Imaging was performed under a Leica SP8 confocal laser scanning microscope.

For wheat germ agglutinin (WGA) staining, the slides were deparaffinized and rinsed three times in PBS and then were incubated for $1 \mathrm{~h}$ at room temperature with a primary antibody against WGA conjugated to FITC (Sigma). The slides were then rinsed three times in PBS, mounted in Vectashield (Vector Labs, CA, USA) and imaged using a Leica SP8 confocal laser scanning microscope, as described above.

\section{Western blotting}

Western blotting assays were performed as previously described [65], with limited modifications. The hearts were homogenized and incubated in RIPA lysis buffer with $1 \mathrm{mM}$ PMSF (phenylmethylsulfonyl fluoride, Beyotime Institute of Biotechnology, Beijing, China). After homogenizing the hearts, $30 \mu \mathrm{g}$ of total protein was loaded onto SDS-PAGE and subsequently blotted onto a nitrocellulose membrane. The membranes were incubated overnight at $4{ }^{\circ} \mathrm{C}$ with primary antibodies against phospho-STAT3 (Tyr705), STAT3 (D3Z2G), phospho-p44/42 MAPK (Erk1/2) (Thr202/Tyr204), p44/42 MAPK (Erk1/2) (137F5), phospho-Akt (Ser473), and Akt and then were washed three times with TBST buffer before adding the secondary antibody. All of the antibodies were acquired from Cell Signaling Technology. The bands were visualized using the enhanced chemiluminescence (ECL) kit (GE Healthcare).

\section{RT-PCR array screening of inflammatory cytokines}

To assess the gene expression profiles of major inflammatory cytokines in the injured hearts of neonatal mice, we performed $\mathrm{RT}^{2}$ profiler PCR arrays (SA Biosciences, Frederick, MD, USA) to identify the potential cytokines that played important roles during the early stages of heart regeneration in neonatal mice. In brief, $1 \mu \mathrm{g}$ of RNA was reverse transcribed with the $\mathrm{RT}^{2}$ first strand kit (Qiagen), followed by qPCR with $\mathrm{RT}^{2}$ SYBR Green ROX qPCR Mastermix (Qiagen). The 96-well plates were processed in the Applied Biosystems 7300 Real Time PCR System. The data were analyzed by using the web-based $\mathrm{RT}^{2}$ profiler PCR array data analysis tool from SABiosciences.

\section{Statistical analysis}

All data are presented as mean \pm SEM. Student's unpaired $t$-test was used for comparisons between two groups. A value of $P<0.05$ was considered statistically significant.

\section{Acknowledgments}

The study was supported by the National Natural Science Foundation of China (Grant No. 81430006, 81500239, 81441010), the National Basic Research Program of China (Program 973; 
2010CB529505), the Fundamental Research Funds for the Central Universities (2012-XHGX02) and the Innovation Fund for Graduates of Peking Union Medical College (Grant No. 2013-1002-44). We sincerely thank Bin Zhou, Lianfeng Zhang, Wei Tao, Xi Wang and Xudong $\mathrm{Wu}$ for the insightful discussions.

\section{References}

1 Ptaszek LM, Mansour M, Ruskin JN, Chien KR. Towards regenerative therapy for cardiac disease. Lancet 2012; 379:933942.

2 Xin M, Olson EN, Bassel-Duby R. Mending broken hearts: cardiac development as a basis for adult heart regeneration and repair. Nat Rev Mol Cell Biol 2013; 14:529-541.

3 Bergmann O, Bhardwaj RD, Bernard S, et al. Evidence for cardiomyocyte renewal in humans. Science 2009; 324:98-102.

4 Senyo SE, Steinhauser ML, Pizzimenti CL, et al. Mammalian heart renewal by pre-existing cardiomyocytes. Nature 2013; 493:433-436.

5 Ali SR, Hippenmeyer S, Saadat LV, Luo L, Weissman IL, Ardehali R. Existing cardiomyocytes generate cardiomyocytes at a low rate after birth in mice. Proc Natl Acad Sci USA 2014; 111:8850-8855.

6 Laflamme MA, Murry CE. Heart regeneration. Nature 2011; 473:326-335.

7 Christoffels VM, Pu WT. Developing insights into cardiac regeneration. Development 2013; 140:3933-3937.

8 Porrello ER, Mahmoud AI, Simpson E, et al. Transient regenerative potential of the neonatal mouse heart. Science 2011; 331:1078-1080.

9 Hassink RJ, Pasumarthi KB, Nakajima H, et al. Cardiomyocyte cell cycle activation improves cardiac function after myocardial infarction. Cardiovasc Res 2008; 78:18-25.

10 Pasumarthi KB, Nakajima H, Nakajima HO, Soonpaa MH, Field LJ. Targeted expression of cyclin D2 results in cardiomyocyte DNA synthesis and infarct regression in transgenic mice. Circ Res 2005; 96:110-118.

11 Soonpaa MH, Koh GY, Pajak L, et al. Cyclin D1 overexpression promotes cardiomyocyte DNA synthesis and multinucleation in transgenic mice. J Clin Invest 1997; 99:2644-2654.

12 Woo YJ, Panlilio CM, Cheng RK, et al. Therapeutic delivery of cyclin A2 induces myocardial regeneration and enhances cardiac function in ischemic heart failure. Circulation 2006; 114:I206-213.

13 Bersell K, Arab S, Haring B, Kuhn B. Neuregulin1/ErbB4 signaling induces cardiomyocyte proliferation and repair of heart injury. Cell 2009; 138:257-270.

14 Heallen T, Morikawa Y, Leach J, et al. Hippo signaling impedes adult heart regeneration. Development 2013; 140:46834690.

15 Xin M, Kim Y, Sutherland LB, et al. Hippo pathway effector Yap promotes cardiac regeneration. Proc Natl Acad Sci USA 2013; 110:13839-13844.

16 Moya IM, Halder G. Discovering the Hippo pathway protein-protein interactome. Cell Res 2014; 24:137-138.

17 Eulalio A, Mano M, Dal Ferro M, et al. Functional screening identifies miRNAs inducing cardiac regeneration. Nature 2012; 492:376-381.

18 Porrello ER, Mahmoud AI, Simpson E, et al. Regulation of neonatal and adult mammalian heart regeneration by the miR15 family. Proc Natl Acad Sci USA 2013; 110:187-192.

19 Mahmoud AI, Kocabas F, Muralidhar SA, et al. Meis1 regulates postnatal cardiomyocyte cell cycle arrest. Nature 2013; 497:249-253.

20 Oyama K, El-Nachef D, MacLellan WR. Regeneration potential of adult cardiac myocytes. Cell Res 2013; 23:978-979.

21 Aurora AB, Porrello ER, Tan W, et al. Macrophages are required for neonatal heart regeneration. J Clin Invest 2014; 124:1382-1392.

22 Frangogiannis NG. The immune system and cardiac repair. Pharmacol Res 2008; 58:88-111.

23 Frangogiannis NG. Regulation of the inflammatory response in cardiac repair. Circ Res 2012; 110:159-173.

24 Marchant DJ, Boyd JH, Lin DC, Granville DJ, Garmaroudi FS, McManus BM. Inflammation in myocardial diseases. Circ Res 2012; 110:126-144.

25 Arslan F, de Kleijn DP, Pasterkamp G. Innate immune signaling in cardiac ischemia. Nat Rev Cardiol 2011; 8:292-300.

26 Nahrendorf M, Swirski FK. Monocyte and macrophage heterogeneity in the heart. Circ Res 2013; 112:1624-1633.

27 Coggins M, Rosenzweig A. The fire within: cardiac inflammatory signaling in health and disease. Circ Res 2012; 110:116125.

28 Nahrendorf M, Swirski FK, Aikawa E, et al. The healing myocardium sequentially mobilizes two monocyte subsets with divergent and complementary functions. J Exp Med 2007; 204:3037-3047.

29 Nahrendorf M, Pittet MJ, Swirski FK. Monocytes: protagonists of infarct inflammation and repair after myocardial infarction. Circulation 2010; 121:2437-2445.

30 Swirski FK, Nahrendorf M, Etzrodt M, et al. Identification of splenic reservoir monocytes and their deployment to inflammatory sites. Science 2009; 325:612-616.

31 Heredia JE, Mukundan L, Chen FM, et al. Type 2 innate signals stimulate fibro/adipogenic progenitors to facilitate muscle regeneration. Cell 2013; 153:376-388.

32 Saclier M, Cuvellier S, Magnan M, Mounier R, Chazaud B. Monocyte/macrophage interactions with myogenic precursor cells during skeletal muscle regeneration. FEBS J 2013; 280:4118-4130.

33 Huang GN, Thatcher JE, McAnally J, et al. C/EBP transcription factors mediate epicardial activation during heart development and injury. Science 2012; 338:1599-1603.

34 Carr KD, Sieve AN, Indramohan M, Break TJ, Lee S, Berg RE. Specific depletion reveals a novel role for neutrophil-mediated protection in the liver during Listeria monocytogenes infection. Eur J Immunol 2011; 41:2666-2676.

35 Daley JM, Thomay AA, Connolly MD, Reichner JS, Albina JE. Use of Ly6G-specific monoclonal antibody to deplete neutrophils in mice. J Leukoc Biol 2008; 83:64-70.

36 Muñoz-Cánoves P, Scheele C, Pedersen BK, Serrano AL. Interleukin-6 myokine signaling in skeletal muscle: a double-edged sword? FEBS J 2013; 280:4131-4148.

37 Cressman DE, Greenbaum LE, DeAngelis RA, et al. Liver failure and defective hepatocyte regeneration in interleukin-6-deficient mice. Science 1996; 274:1379-1383.

38 Aurora AB, Olson EN. Immune modulation of stem cells and regeneration. Cell Stem Cell 2014; 15:14-25. 
39 Przybyt E, Krenning G, Brinker M, Harmsen MC. Adipose stromal cells primed with hypoxia and inflammation enhance cardiomyocyte proliferation rate in vitro through STAT3 and Erk1/2. J Transl Med 2013; 11:39.

40 Harrison DA. The Jak/STAT pathway. Cold Spring Harb Perspect Biol 2012; 4:a011205.

41 Fang Y, Gupta V, Karra R, Holdway JE, Kikuchi K, Poss KD. Translational profiling of cardiomyocytes identifies an early Jak1/Stat3 injury response required for zebrafish heart regeneration. Proc Natl Acad Sci USA 2013; 110:13416-13421.

42 Zhang C, Li Y, Wu Y, Wang L, Wang X, Du J. Interleukin-6/ signal transducer and activator of transcription 3 (STAT3) pathway is essential for macrophage infiltration and myoblast proliferation during muscle regeneration. J Biol Chem 2013; 288:1489-1499.

43 Joe AW, Yi L, Natarajan A, et al. Muscle injury activates resident fibro/adipogenic progenitors that facilitate myogenesis. Nat Cell Biol 2010; 12:153-163.

44 Kami K, Senba E. Localization of leukemia inhibitory factor and interleukin-6 messenger ribonucleic acids in regenerating rat skeletal muscle. Muscle Nerve 1998; 21:819-822.

45 Kami K, Senba E. In vivo activation of STAT3 signaling in satellite cells and myofibers in regenerating rat skeletal muscles. J Histochem Cytochem 2002; 50:1579-1589.

46 Aldeguer X, Debonera F, Shaked A, et al. Interleukin-6 from intrahepatic cells of bone marrow origin is required for normal murine liver regeneration. Hepatology 2002; 35:40-48.

47 Taub R. Hepatoprotection via the IL-6/Stat3 pathway. J Clin Invest 2003; 112:978-980.

48 Li W, Liang X, Kellendonk C, Poli V, Taub R. STAT3 contributes to the mitogenic response of hepatocytes during liver regeneration. J Biol Chem 2002; 277:28411-28417.

49 Andersen DC, Ganesalingam S, Jensen CH, Sheikh SP. Do neonatal mouse hearts regenerate following heart apex resection? Stem Cell Rep 2014; 2:406-413.

50 Bryant DM, O'Meara CC, Ho NN, Gannon J, Cai L, Lee RT. A systematic analysis of neonatal mouse heart regeneration after apical resection. J Mol Cell Cardiol 2015; 79:315-318.

51 Konfino T, Landa N, Ben-Mordechai T, Leor J. The type of injury dictates the mode of repair in neonatal and adult heart. $J$ Am Heart Assoc 2015; 4:e001320.

52 Darehzereshki A, Rubin N, Gamba L, et al. Differential regenerative capacity of neonatal mouse hearts after cryoinjury. Dev Biol 2015; 399:91-99.

53 Polizzotti BD, Ganapathy B, Walsh S, et al. Neuregulin stimulation of cardiomyocyte regeneration in mice and human myocardium reveals a therapeutic window. Sci Transl Med 2015; 7:281ra245.

54 Mahmoud AI, Porrello ER, Kimura W, Olson EN, Sadek HA. Surgical models for cardiac regeneration in neonatal mice. Nat Protoc 2014; 9:305-311.
55 O'Meara CC, Wamstad JA, Gladstone RA, et al. Transcriptional reversion of cardiac myocyte fate during mammalian cardiac regeneration. Circ Res 2015; 116:804-815.

56 Sadek HA, Martin JF, Takeuchi JK, et al. Multi-investigator letter on reproducibility of neonatal heart regeneration following apical resection. Stem Cell Rep 2014; 3:1.

57 Rui L, Yu N, Hong L, et al. Extending the time window of mammalian heart regeneration by thymosin beta 4 . J Mol Cell Cardiol 2014; 18:2417-2424.

58 Haubner BJ, Adamowicz-Brice M, Khadayate S, et al. Complete cardiac regeneration in a mouse model of myocardial infarction. Aging (Albany NY) 2012; 4:966-977.

59 Kopf M, Baumann H, Freer G, et al. Impaired immune and acute-phase responses in interleukin-6-deficient mice. Nature 1994; 368:339-342.

60 Moh A, Iwamoto Y, Chai G-X, et al. Role of STAT3 in liver regeneration: survival, DNA synthesis, inflammatory reaction and liver mass recovery. Lab Invest 2007; 87:1018-1028.

61 Sohal DS, Nghiem M, Crackower MA, et al. Temporally regulated and tissue-specific gene manipulations in the adult and embryonic heart using a tamoxifen-inducible Cre protein. Circ Res 2001; 89:20-25.

62 Egawa G, Osawa M, Uemura A, Miyachi Y, Nishikawa S. Transient expression of ephrin b2 in perinatal skin is required for maintenance of keratinocyte homeostasis. J Invest Dermatol 2009; 129:2386-2395.

63 Sander LE, Sackett SD, Dierssen U, et al. Hepatic acutephase proteins control innate immune responses during infection by promoting myeloid-derived suppressor cell function. $J$ Exp Med 2010; 207:1453-1464.

64 Liao XD, Wang XH, Jin HJ, Chen LY, Quan C. Mechanical stretch induces mitochondria-dependent apoptosis in neonatal rat cardiomyocytes and $\mathrm{G} 2 / \mathrm{M}$ accumulation in cardiac fibroblasts. Cell Res 2004; 14:16-26.

65 Han P, Zhou X-H, Chang N, et al. Hydrogen peroxide primes heart regeneration with a derepression mechanism. Cell Res 2014; 24:1091-1107.

(Supplementary information is linked to the online version of the paper on the Cell Research website.)

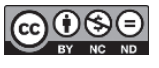

This work is licensed under a Creative Commons Attribution-NonCommercial-NoDerivs 4.0 Unported License. The images or other third party material in this article are included in the article's Creative Commons license, unless indicated otherwise in the credit line; if thematerial is not included under the Creative Commons license, users will need to obtain permission from the license holder to reproduce the material. To view a copy of this license, visit http://creativecommons.org/licenses/by-nc-nd/4.0/ 